\title{
ON BIEBERBACH EILENBERG FUNCTIONS
}

BY DOV AHARONOV

Communicated by Wolfgang H. Fuchs, July 18, 1969

I. Introduction. In this paper we bring the following two results:

Suppose that $F(z)=b_{1} z+b_{2} z^{2}+, \cdots$ is a B.E. function (i.e. $F(z)$ is regular in the unit circle, $F(z) F(\zeta) \neq 1$ for any $|z|,|\zeta|<1$ and $F(0)=0)$. Then we have

$$
\sum_{k=1}^{\infty}\left|b_{k}\right|^{2} \leqq 1
$$

This result contains, of course, the result

$$
\left|b_{n}\right| \leqq 1, \quad n=1,2, \cdots
$$

which was conjectured by Rogosinsky [8] and was solved about ten years later by Lebedev and Milin [5].

The second result deals with univalent B.E. function $F(z)$ $=b_{1} z+b_{2} z^{2}+\cdots$. For such function we have the following

$$
\left|b_{n}\right| \leqq e^{-c / 2}(n-1)^{-1 / 2}, \quad n=2,3, \cdots,
$$

where $c$ is Euler constant.

This result is sharp in order of magnitude and the constant cannot be improved to be better than $e^{-1 / 2}$.

II. The results of Lebedev and Milin. Lebedev and Milin found [6], [7] some important results concerning coefficients of exponential functions which we quote here.

Lemma 1. Let $A_{1}, A_{2}, A_{3}, \cdots$ be an infinite sequence of arbitrary complex numbers such that $\sum_{k=1}^{\infty} k\left|A_{k}\right|^{2}<\infty$. Then for $\exp \sum_{k=1}^{\infty} A_{k} z^{k}$ $=\sum_{k=0}^{\infty} D_{k} \mathbf{s}^{k}$ we have

$$
\sum_{k=0}^{\infty}\left|D_{k}\right|^{2} \leqq \exp \sum_{k=1}^{\infty} k\left|A_{k}\right|^{2}
$$

with equality only in the case $A_{k}=\rho^{k} \eta^{k} / k, k=1,2, \ldots$ where $0 \leqq \rho<1$ $|\eta|=1$.

AMS Subject Classifications. Primary 3043, 3009; Secondary 3042, 3065.

Key Words and Phrases. Regular functions, Bieberbach-Eilenberg functions, Milin-Lebedev Lemmas, Schiffer-Garabedian inequalities, pairs of regular functions, Grunsky inequalities. 
Lemma 2. Let $\left\{A_{k}\right\}$ and $\left\{D_{k}\right\}$ be defined as in Lemma 1 (without the limitation $\left.\sum_{k=1}^{\infty} k\left|A_{k}\right|^{2}<\infty\right)$. Then

$$
\left|D_{n}\right|^{2} \leqq \exp \left(\sum_{k=1}^{n} k\left|A_{k}\right|^{2}-\sum_{k=1}^{n} 1 / k\right), \quad n=1,2, \cdots
$$

with equality only in the case $A_{k}=\eta^{k} / k$ for $k=1,2, \cdots, n$ and $|\eta|=1$.

III. Schiffer-Garabedian inequalities. We quote here a theorem of Garabedian and Schiffer [1]:

Lemma 3. Suppose that $F(z)$ is a univalent B.E. function. Then we have for

$$
\begin{aligned}
& \log \frac{F(z)-F(\zeta)}{(z-\zeta)[1-F(z) F(\zeta)]}=\sum_{n, m=0}^{\infty} \gamma_{n m} z^{n} \zeta^{m}, \\
& \operatorname{Re}\left\{\sum_{n, m=0}^{N} \lambda_{n} \lambda_{m} \gamma_{n m}\right\} \leqq \sum_{n=1}^{N} \frac{\left|\lambda_{n}\right|^{2}}{n}
\end{aligned}
$$

where $\lambda_{0}, \lambda_{1}, \lambda_{2}, \cdots, \lambda_{N}$ is a finite sequence of complex constants with $\lambda_{0}$ real.

This remarkable result was proved first in [1] by variational methods. Later the result was proved in [3] by area methods. We note that in [1] the result was formulated in a different manner.

IV. Coefficients of B.E. functions. From Lemma 3 we deduce immediately the following:

$$
\sum_{k=1}^{\infty} k\left|\gamma_{k 0}\right|^{2} \leqq \log \frac{1}{\left|F^{\prime}(0)\right|^{2}} .
$$

(Indeed from Lemma 3 we have

$$
\lambda_{0}^{2} \operatorname{Re}\left\{\log F^{\prime}(0)\right\}+2 \lambda_{0} \operatorname{Re}\left\{\sum_{n=1}^{N} \lambda_{n} \gamma_{n 0}\right\} \leqq 2 \sum_{n=1}^{N} \frac{\left|\lambda_{n}\right|^{2}}{n} .
$$

By substitution $\lambda_{0}=2, \lambda_{n}=n \bar{\gamma}_{n 0}$ we get (8).)

We are now in a position to prove

THeOREM 1. Let $F(z)=b_{1} z+b_{2} z^{2}+\cdots$ be a B.E. function; then (1) follows.

Proof. By substituting $\zeta=0$ in (6) we have

(9) $\log \frac{F(z)}{z}=\sum_{n=0}^{\infty} \gamma_{n 0} z^{n}, \frac{F(z)}{z F^{\prime}(0)}=\exp \left(\sum_{k=1}^{\infty} \gamma_{0 k} z^{k}\right)=\sum_{k=1}^{\infty} \frac{b_{k}}{F^{\prime}(0)} z^{k-1}$. 
By Lemma 1 and (8) we get

$$
\sum_{k=1}^{\infty} \frac{\left|b_{k}\right|^{2}}{\left|F^{\prime}(0)\right|^{2}} \leqq \exp \left(\sum_{k=1}^{\infty} k\left|\gamma_{0 k}\right|^{2}\right) \leqq \frac{1}{\left|F^{\prime}(0)\right|^{2}} .
$$

So our theorem follows for univalent B.E. function. The result is generalized to the general class by the principle of subordination [2, pp. 424-425], [9].

REMARK 1. The result is sharp for the B.E. function $F(z)=z^{n}, n$ $=1,2, \cdots$ and also for Jenkin's functions [4]

$$
F(z)=\frac{\left(1-r^{2}\right)^{1 / 2} z}{1+i r z}, \quad 0 \leqq r<1 .
$$

REMARK 2. Jenkin's result [4]

$$
F(z) \leqq|z| /\left(1-|z|^{2}\right)^{1 / 2}
$$

follows easily from Theorem 1.

TheOREM 2. Let $F(z)=b_{1} z+b_{2} z^{2}+\cdots$ be a univalent B.E. function. Then we have

$$
\left|b_{n}\right|<e^{-c / 2}(n-1)^{-1 / 2}, \quad n=2,3, \cdots
$$

where $c$ is Euler constant.

Proof. By Lemma 2 and (8), (9) we have

$$
\begin{aligned}
& \frac{\left|b_{n}\right|^{2}}{\left|F^{\prime}(0)\right|^{2}} \leqq \exp \left(\sum_{k=1}^{n-1} k\left|\gamma_{0 k}\right|^{2}-\sum_{k=1}^{n-1} 1 / k\right) \leqq \frac{\exp \left(-\sum_{k=1}^{n-1} 1 / k\right)}{\left|F^{\prime}(0)\right|^{2}} \\
& n=2,3, \cdots \text {. }
\end{aligned}
$$

So $\left|b_{n}\right|^{2}<e^{-c}(n-1)^{-1}$ which is another form of our theorem. For Jenkin's functions (11) we have $\left|b_{n}\right|^{2}=\left(1-r^{2}\right) r^{2(n-1)}$. If $1-r^{2}$ $=1 /(n-1)$ we have

$$
\left|b_{n}\right|^{2}=\frac{1}{n-1}\left(1-\frac{1}{n-1}\right)^{n-1} \sim \frac{1}{e(n-1)} .
$$

So the order of magnitude is the best possible and the argument for the constant also follows.

\section{REFERENCES}

1. P. R. Garabedian and M. Schiffer, The local maximum theorem for the coefficients of univalent functions, Arch. Rational Mech. Anal. 26 (1967), 1-32. MR 37 \#1584. 
2. E. Hille, Analytic function theory. Vol. II: Introduction to Higher Mathematics, Ginn, Boston, Mass., 1962. MR 34 \#1490.

3. J. A. Hammel and M. Schiffer, Coefficient inequalities for Bieberbach-Eilenberg functions, Arch. Rational Mech. Anal. 32 (1969), 87-99.

4. J. A. Jenkins, On Bieberbach-Eilenberg functions, Trans. Amer. Math. Soc. 76 (1954), 389-396. MR 16, 24.

5. N. A. Lebedev and I. M. Milin, On the coefficients of certain classes of analytic functions, Mat. Sb. 28 (70) (1951), 359-400. (Russian) MR 13, 640.

6. —_, An inequality, Vestnik Leningrad. Univ. 20 (1965), no. 19, 157-158. (Russian) MR $32 \# 4248$.

7. I. M. Milin, The coefficients of schlicht functions, Dokl. Akad. Nauk SSSR 176 (1967), 1015-1018 = Soviet Math. Dokl. 8 (1967), 1255-1258. MR 36 \#5328.

8. W. W. Rogosinsky, On a theorem of Bieberbach-Eilenberg, J. London Math. Soc. 14 (1939), 4-11.

9. - On the coefficients of subordinate functions, Proc. London Math. Soc. (2) 48 (1943), 48-82. MR 5, 36.

Technion Israel Institute of Technology, Haifa, Israel 